2

3

5 Jonker $^{1,4}$

6

7 6

\title{
Contrasting context-dependence of familiarity and kinship in animal social networks
}

Ralf HJM Kurvers ${ }^{1,2, *}$, Vena MAP Adamczyk ${ }^{1}$, Robert HS Kraus ${ }^{1,3}$, Joseph I Hoffman ${ }^{4}$, Sipke E van Wieren ${ }^{1}$, Henk $\mathrm{P}$ van der Jeugd ${ }^{5}$, William Amos $^{6}$, Herbert HT Prins ${ }^{1,7}$, Rudy M

7 Resource Ecology Group, Wageningen University, Droevendaalsesteeg 3a, 6708 PB Wageningen, the Netherlands.

${ }^{2}$ Leibniz-Institute of Freshwater Ecology and Inland Fisheries. Müggelseedamm 310, 12587 Berlin, Germany.

${ }^{3}$ Conservation Genetics Group, Senckenberg Research Institute and Natural History Museum Frankfurt, Clamecystr. 12, 63571, Gelnhausen, Germany.

${ }^{4}$ Department of Animal Behaviour, University of Bielefeld, Morgenbreede 45, 33615 Bielefeld, Germany.

5 Vogeltrekstation - Dutch Centre for Avian Migration \& Demography, NIOO-KNAW, Wageningen, The Netherlands.

${ }^{6}$ Department of Zoology, University of Cambridge, Downing Street, Cambridge, CB2 3EJ, United Kingdom.

${ }^{7}$ School for Biological and Conservation Science, University of Kwazulu-Natal, Westville Campus, Private Bag X54001, Durban, 4000, South Africa.

* Corresponding author: Ralf HJM Kurvers. Leibniz-Institute of Freshwater Ecology and Inland Fisheries. Müggelseedamm 310, 12587 Berlin, Germany. Email address: ralf.kurvers@igb-berlin.de

Word count: 8,454 
The social structure of a population is a crucial element of an individual's environment, fundamentally impacting the transfer of genes, information and diseases. A central question in social network analysis is how different traits affect associations within populations. However, previous studies of animal social networks have typically focused on a single predictor or stage in the life cycle whereas social interactions within populations are known to be dynamic and not fixed through time and/or context. Relatively few animal network studies have explored how individual traits impact decisions across different ecologically relevant contexts. Here we collected detailed behavioural data (personality, dominance, familiarity) and high resolution genetic data from a flock of 43 captive barnacle geese to understand how these traits affect association patterns in two different evolutionary and ecologically highly relevant contexts: foraging and mate choice. Using a novel analytical framework for nodelabel permutations, we show that barnacle geese preferentially associate with close kin and other individuals familiar from earlier in life when foraging, but select for partners that are unfamiliar during mate choice. We found no effect of either personality or dominance on foraging associations or mate choice. Our study shows how using social network analysis can increase our understanding of the drivers behind population structure (in our case kin selection and inbreeding avoidance). Moreover, our study demonstrates that social networks can be largely determined by long-term processes, in particular early-life familiarity.

Keywords: barnacle goose, Branta leucopsis, food sharing, inbreeding avoidance, kin recognition, kinship, sexual selection 
The social structure of a population is a crucial element of an individual's environment and can have important consequences for the ecology and behaviour of individuals. For example, it can fundamentally impact migration patterns (Guttal \& Couzin 2010), cooperation (Croft et al. 2006) and the transmission of diseases (Cross et al. 2004) and information (Couzin et al. 2005; Aplin et al. 2012). Social network analysis allows the investigation of the fine social structure of animal groups and currently one of the important questions is how individual traits affect the structure of animal groups (Croft et al. 2008; Wey et al. 2008; Whitehead 2008). Here, data are collected across all pairwise comparisons among individuals, allowing both the strongest and most frequently interacting pairs to be identified as well as revealing correlations between different variables. With this approach, evidence has been generated for the effects of a number of different traits on social structure, most of which are to be expected, such as genetic relatedness, familiarity, personality, dominance, age, sex and morphological traits [e.g. (Krause et al. 2007; Croft et al. 2008; Pike et al. 2008; Krause et al. 2009; Farine et al. 2012)].

Social networks (or associations among individuals) are dynamic and not fixed through context (Wiszniewski et al. 2012; Hobson et al. 2013). When deciding with whom to interact, individuals may use different criteria dynamically and incorporate information from past experiences (Sih et al. 2009). However, the temporal and contextual elements of association preferences have been little studied and relatively few studies have explored how individual traits impact decisions across different ecologically relevant contexts [but see (Flack et al. 2006; Lusseau et al. 2011; Hirsch et al. 2012; Madden et al. 2012; Wey \& Blumstein 2012)]. Likewise, few studies have addressed how experiences early in life affect association patterns of animals in social networks later in life (Hobson et al. 2013). To address these shortfalls requires sets of observations that follow the same group of individuals over longer time windows, preferably their entire lives, as well as detailed data collection across 
contexts, to define how individual traits and early life experiences affect association patterns across time and ecological context.

Here we studied how early life familiarity, genetic relatedness, boldness and dominance affect the association patterns between barnacle geese Branta leucopsis in two different, evolutionary and ecologically highly relevant, contexts: foraging and mate choice. Barnacle geese offer an excellent model system for the study of social interactions and networks. As a species they are large, easy to observe and their key life history traits are likely modified by social interactions. For this study we used a population of 43 captive geese. In respect of foraging associations we expected to observe (1) positive assortment for genetic relatedness and familiarity, as this might incur inclusive fitness benefits (Mathot \& Giraldeau 2010) and a stronger effect in females than in males, because natal philopatry is female-biased in this species (Anderholm et al. 2009); (2) negative assortment for boldness, since bolder barnacle geese are known to arrive first at food patches, whereas shyer individuals tend to follow more frequently (Kurvers et al. 2011; Kurvers et al. 2012b); (3) negative assortment for dominance, since aggression between animals of disparate dominance rank can be lower compared to animals of similar dominance rank (Cote 2000; Forkman \& Haskell 2004). In respect of mate choice, we expected to observe (4) disassortative mating with respect to genetic relatedness to avoid inbreeding; (5) assortative mating with respect to familiarity based on earlier findings in this species (Choudhury \& Black 1994; Black \& Owen 1995). We had no a priori expectations in respect of dominance or boldness (Schuett et al. 2010). 


\section{METHODS}

\section{Study subjects}

We procured two mixed-sex groups (hereafter called 'familiarity groups', $N=21$ and 22 , respectively) of barnacle geese, which were housed separately in an outdoor aviary at the Netherlands Institute of Ecology (NIOO) in Heteren, the Netherlands. The two groups were visually but not acoustically isolated. Each aviary $(12 \times 15 \mathrm{~m})$ consisted of bare soil and a large pond $(6 \times 1 \mathrm{~m})$ with continuously flowing water for bathing and drinking. Geese were fed ad libitum with a mixture of grains and pellets and occasionally grass. All geese were captive-born, wing-clipped and fitted with uniquely coded white-coloured leg rings for identification. Geese arrived in November 2007 and most individuals (39 out of 43) were born in 2007 meaning that they were approximately 5 months old when they arrived. The two groups had different origins implying that individuals with high genetic relatedness were only present within each familiarity group but not between groups (see Results). We constructed a familiarity matrix for all geese in which each pair received either a 0 (not from the same familiarity group) or a 1 (from the same familiarity group). Geese lived for approximately 1.5 years in these familiarity groups [during which they participated in other experiments (Kurvers et al. 2009; Kurvers et al. 2012a)] before the social network observations started.

All experiments were approved by the animal ethical committee ("Dier Experimenten Commissie") of both the Royal Netherlands Academy of Arts and Sciences (KNAW) and the Wageningen University [protocol numbers: 2010008.b (blood sampling), 2008107.a and 2008094.b (personality tests)]. Geese were procured from a waterfowl breeding farm (Kooy and Sons, 't Zand, the Netherlands) and after the experiments geese were returned to the breeding farm. 


\section{Foraging associations}

Social network observations were conducted in the home aviaries (12 x $15 \mathrm{~m})$ when geese were circa two years of age. Two weeks before, the geese were separated into two single-sex flocks, allowing the study of sex-specific factors that may contribute to association preferences while avoiding any confounding inter-sexual interactions. We thus performed our observations on one group consisting of all males, and one group consisting of all females. Geese were sexed by HPJ using visual inspection of sexual organs in the cloaca (group 1: 13 females, 8 males; group 2: 7 females, 15 males). Foraging associations were studied on five grass patches $(40 \times 20 \mathrm{~cm}, 1.5 \mathrm{~m}$ apart $)$ along one side of the aviary and were refreshed twice a day to minimize an effect of depletion. Other food sources were removed during the observations. Each single-sex group was observed $(9.00-13.00 \mathrm{~h})$ for 15 days (females: 22 June - 12 July 2009; males: 13 - 30 July 2009) by the same observer (VMAPA). The presence of all individuals on the patches was recorded every four minutes. This optimal interval was determined through test studies to be longer than the mixing interval among individuals (Croft et al. 2008), with group composition seldom (females: 5.9\%, males 7.9\%) being the same in consecutive records. Travel time between the two outer patches was less than a minute, substantially less than the recording frequency. Observations were occasionally interrupted for ten minutes to allow resettling following external disturbance. Since patch size $(40 \times 20 \mathrm{~cm})$ and group size $($ mean females $=1.9$, range $1-5 ;$ mean males $=2.0$, range $1-5)$ were both small, we assumed that animals grazing on the same patch during a sampling period were associating, a method known as gambit of the group (Whitehead 2008; Franks et al. 2010). For each group we derived an association matrix using the Simple Ratio Index (SRI) in SOCPROG (Whitehead 2009). SRI measures the proportion of times two individuals were seen together out of the total number of times those individuals were observed, thereby controlling for inter-individual differences in the total number of sightings. SRI tends to be a 
highly effective measure of association as long as there are no large sampling biases (Ginsberg \& Young 1992). Since all our observations were performed within aviaries in which all individuals feeding on the patches could be easily identified, we do not expect such a sampling bias.

\section{Mate choice}

To investigate which individual traits contribute to mate choice, we placed all geese together in one flock in January 2010. Most geese were around 2.5 years of age, approximating the average age of final pair formation in barnacle geese (Choudhury \& Black 1994; van der Jeugd \& Blaakmeer 2001). In June 2010, we scored which males and females formed a pair bond $(N=20)$. To confirm that these bonds were stable and not merely mate sampling behaviour [so called 'trial liaisons' (Choudhury \& Black 1993; van der Jeugd \& Blaakmeer 2001)] we kept the flock as one group throughout the whole year and scored the pair bonds again in December 2010. All twenty of the pair bonds that were initially identified remained unchanged, indicating that these bonds were highly stable over time. We used the data on pair formation to construct a 'pair matrix' in which each possible dyad was classified either as paired (1) or unpaired (0).

\section{Genetic relatedness}

To separate the effects of familiarity and genetic relatedness, we quantified the degree of genetic relatedness between each pair of individuals by means of a high resolution 374 single nucleotide polymorphism (SNP) marker set specifically designed for the barnacle goose (Jonker et al. 2012). We took a small blood sample (approx. $1 \mathrm{ml}$ ) from each individual from the brachial vein, which was preserved in ethanol. Entire genomic DNA was subsequently isolated using the Gentra Systems Puregene DNA purification kit. Genotyping was conducted 
with Vera Code assays on an Illumina BeadXpress as described in Kraus et al. (2011). Pairwise genetic relatedness $(r)$ was calculated using the program Coancestry version 1 (Wang 2011). To determine which relatedness estimator was the best for our type of data, we used the empirical SNP allele frequencies of our experimental group and simulated 500 dyads of geese of varying relatedness coefficients. Meeting real conditions as closely as possible we simulated mainly unrelated dyads (430 in total) but also dyads of close familial relationships, namely 30 dyads of full sibs $(r=0.5), 10$ dyads of half-sibs $(r=0.25)$ and 30 dyads of first cousins $(r=0.125)$. Based on this trial we found the maximum-likelihood estimator of Milligan (2003) to perform best and used it for the final estimation of $r$. This produced a strong correlation with expected values of $r\left(r^{2}=0.9\right.$; analysis of the simulated data set carried out with default settings). Subsequently, all pairwise relatedness values of the experimental geese were obtained from Coancestry with standard settings to construct a genetic relatedness matrix.

\section{Boldness}

We assessed the boldness level of individuals by performing novel object tests (see for details (Kurvers et al. 2009)). After habituating individuals to an experimental arena ( 9 x $3 \mathrm{~m}) \mathrm{a}$ novel object was presented in the middle for $10 \mathrm{~min}$ and the minimal distance reached between the goose and the novel object was recorded, as well as the approach latency. Each individual was tested twice within a two month period (November-December 2008) (Kurvers et al. 2009; 2010) using as novel objects either a green plastic mat (test 1) or a brown deeppile rug (test 2). Geese were approximately 1.5 years old during these tests and all the novel object tests were conducted in social isolation. We calculated principal components (PCs) of the minimal distance to the novel object and the approach latency for each test as the novel object score. PC1 explained $87 \%$ and $90 \%$ of the variation for test one and test two, 
respectively. The correlations of both the minimal distance and the approach latency with PC1 were negative, implying that high values of PC1 correspond to bolder individuals. To determine the repeatability of novel object scores, we calculated the mean squares from a oneway analysis of variance (ANOVA) with individual fitted as a main effect. Repeatability was calculated following Lessells and Boag (1987) and its standard error following Becker (1984). The resulting repeatability value was high (0.82), indicating that individuals differed consistently in their boldness scores. We averaged the two novel object scores of each individual to arrive at a single measure of boldness. We then derived a boldness distance matrix by calculating the distance in boldness between each pair.

\section{Dominance}

Dominance hierarchies in each single sex flock were generated from all recorded agonistic interactions, defined as a direct confrontation between two geese, ranging from threats with lowered head and neck to active chases with flapping wings. Winners and losers were identified when one pair member showed active avoidance behaviour. Dominance data were collected in the same period as the sampling of the foraging associations. We scored a total of 1429 interactions in the female group and 2844 interactions in the male group. Since the number of unknown relationships was small (females: $4.2 \%$; males: $0.4 \%$ ), we were able to construct full dominance matrices, thereby minimizing inconsistencies (De Vries 1998), rather than using simple dominance scores. Kendall's linearity index, Landau's index and the corrected index of the sociometric matrix were high for both sexes (females: $K=0.64, h=$ $0.64, h^{\prime}=0.65$, males: $K=0.83, h=0.83, h^{\prime}=0.83$ ), allowing the use of a linear order to rank the individuals. Females were ranked from 1 (most subordinate) to 20 (most dominant) and males similarly from 1 to 23 . From these ranks, we derived a dominance distance matrix, 
indicating how (dis)similar each pair of individuals was in terms of their dominance. We constructed separate matrices for females and males.

For the analysis of mate choice, we did not use dominance rank data derived from the mixed-sex flock, since pair formation affects the dominance hierarchy in geese, with pairs dominating singletons (Black \& Owen 1989; van der Jeugd \& Blaakmeer 2001). Moreover, both pair members obtain similar dominance ranks because they operate as a social unit (Black \& Owen 1989). In agreement with previous work on this species (Kurvers et al. 2009) dominance and boldness were not correlated (females: $r_{s}=0.03, p=0.89$; males: $r_{s}=0.15, p=$ $0.49)$.

\section{Statistical analysis}

To analyse our data we developed a novel node-label permutation test with general(ized) linear models fitted to linearized similarity / dissimilarity matrices. We used the SRI matrix as the dependent variable and fitted as independent variables the familiarity matrix, the genetic relatedness matrix, the boldness distance matrix and the dominance distance matrix. The significance of each independent variable was calculated by permutation: randomly scrambling the row orders of the matrix of that variable while keeping all others constant and counting the number of times the AIC (Akaike Information Criterion) of the scrambled dataset was lower than that of the unscrambled data using general linear models. For the unscrambled general linear models, the residuals were visually inspected for normality. The columns were scrambled in the same order as the rows to maintain the inherent properties of the matrix which prevents impossible matrices being generated. We refer to this method as MRMPA (Multiple Regression Matrix-Permutation using AIC). Scrambling was repeated 10,000 times. We fitted separate models for females and males. To calculate the effect sizes and standard errors of the variables in the models we linearized the matrices and extracted a 
random sample ( $\mathrm{n}=$ number of rows, sampling with replacement) of all rows from these columns, and computed the estimates and standard errors of the model. This was repeated 10,000 times, after which we calculated the mean estimate and the mean standard error for each of the variables. All scrambling procedures and linear models were performed in $\mathrm{R}$ version 2.14.2.

To compare our results with current methods used in social network analysis we also used the Multiple Regression Quadratic Assignment Procedures (MRQAP) (Krackhardt 1988; Dekker et al. 2007) with the double Dekker semi-partialing permutation method in UCINET version 6.414. MRQAP calculates partial matrix regression coefficients for a response matrix on several explanatory matrices and then uses a large number of random node-label permutations within matrices [i.e., bootstrapping (Efron \& Tibshirani 1994)] to generate a sampling distribution and assign $p$-values. From UCINET we obtained $p$-values together with mean estimates and standard errors for all variables, and compared these to the results obtained by MRMPA.

To analyse the determinants of mate choice we used the (binary) mate pair matrix as the dependent variable and fitted as independent variables the familiarity matrix, the genetic relatedness matrix and the boldness distance matrix. The significance of each independent variable was calculated using a similar procedure as described above (MRMPA) but with two additional restrictions. First, rows (and likewise columns) were only scrambled within sex to avoid the formation of same sex pairs in the scrambled matrix. Second, we used generalized linear models (GzLM) with a logit-link function. The bootstrap procedure for obtaining the estimates and standard errors of the model was more complicated because of the binomial data structure and because only few pairs (20) were present out of the total possible number of pairs (946). Therefore, we sampled 5,000 times from the possible rows (with replacement). This was repeated 10,000 times, after which we calculated the mean estimate and the mean 
standard error for all variables. However, the use of MRQAP for the mate choice analysis is problematic. First, it does not allow scrambling within certain subsets of rows and columns (i.e., within sex in our case). Second, it is not clear how robustly MRQAP deals with binary variables (Dekker et al. 2007). Therefore, we did not use MRQAP to analyse pair formation.

\section{RESULTS}

\section{Genetic relatedness}

The average pairwise relatedness among all of the dyads was $0.035 \pm 0.094$ SD (range: 0 0.636). The majority of dyads were unrelated: 755 out of a total of 903 dyads had relatedness $<0.05$; only 47 dyads had relatedness $>0.15$ and these dyads were only present within familiarity groups. Mean pairwise relatedness was elevated within familiarity groups (mean \pm $\mathrm{SD}=0.062 \pm 0.12$; range: $0-0.636$ ) in comparison to dyads representing different familiarity groups (mean $\pm \mathrm{SD}=0.009 \pm 0.020$; range: $0-0.149$ ) supporting the assumption that highly related individuals were only present within familiarity groups (Fig. 1).

\section{Foraging associations}

Average SRI within the female group was $0.040 \pm 0.059$ SD (range $0-0.44$ ), with most females having relatively few strong and many weak connections (Fig. 2a, Appendix 1). Female geese associated significantly more with individuals from the same familiarity group (MRMPA: $P<0.0001$, Table 1, Fig. 3a, 4). A strong, highly significant association was found between genetic relatedness and association strength (MRMPA: $p<0.0001$, Table 1, Fig. 3a, 4). In contrast, boldness and dominance were both uncorrelated with association strength 
(MRMPA: boldness: $P=0.17$; dominance: $P=0.90$; Table 1, Fig. 4). MRQAP returned virtually identical results (see Table 1).

Average SRI within the male group was $0.034 \pm 0.057 \mathrm{SD}$ (range $0-0.50$ ), with most individuals again having relatively few strong associations (Fig. 2b, Appendix 2). Male geese associated significantly more with individuals from the same familiarity group (MRMPA: $P=$ 0.043, Table 1, Fig. 3b, 4). A significant positive relationship was again found between genetic relatedness and association strength (MRMPA: $P=0.002$, Table 1, Fig. 3b, 4) although this was weaker than that found for females. Similarly, boldness and dominance were unrelated to association strength (MRMPA: boldness: $P=0.88$; dominance: $P=0.52$; Fig. 4). MRQAP returned similar results (see Table 1).

\section{Mate choice}

Individuals chose a mate significantly more often from the unfamiliar than the familiar group (MRMPA: $P=0.030$, Table 1, Fig. 4), with 15 out of 20 pair bonds being between individuals of different familiarity groups. There was no effect of genetic relatedness or boldness on mate choice (see Table 1).

\section{DISCUSSION}

In this study we have investigated how early life familiarity, genetic relatedness, boldness and dominance affect the strength of association between barnacle goose individuals in two different, evolutionary and ecologically highly relevant, contexts: foraging and mate choice. For this we combined an experimental approach with detailed behavioural observations, high resolution genetic data and a novel analytical framework. We show contrasting context- 
dependence of familiarity and kinship in social networks: when foraging, barnacle geese preferentially associate with individuals that are genetically related and familiar from earlier in their life, but when choosing a mate, they select against familiarity. Traits can thus drive associations in opposing directions depending on the ecological context. Studies looking at multiple types of interaction networks are currently emerging (Flack et al. 2006; Lusseau et al. 2011; Hirsch et al. 2012; Madden et al. 2012; Wey \& Blumstein 2012) and our results suggest that generalization across contexts can be problematic. This can have important consequences, for example for predicting disease transmission during different life stages of an individual (Drewe 2010). Furthermore, group members interact with one another in multiple social or behavioural contexts and interactions in one context are not independent of interactions in others but there can be carry over effects between contexts (Flack et al. 2006; Sih et al. 2009). This concept of multidimensional social networks (Lusseau et al. 2011) has thus far received little attention. Likewise, there is very little understanding of how early life experiences shape social network structure later in life. This is remarkable since the social networks of many species studied in the context of animal social networks consist of individuals that know each other for a long time (and/or are genetically related). Here we show that interactions early in life can have profound consequences on social network structure later in life (both in a foraging and mate choice context), highlighting the importance of approaches that integrate early life experiences in studies of animal social networks.

\section{Kin selection, kin recognition and inbreeding}

Hamilton's rule (Hamilton 1964) predicts kin-favoured food sharing when the costs of sharing resources are low. However, if there are high costs of food-sharing, for instance due to strong exploitative competition, individuals should avoid kin so that the burden of resource competition is placed on non-kin. Several recent studies indeed highlight the importance of 
resource availability on the stability of social relationships in social networks (Henzi et al. 2009; Foster et al. 2012; Holekamp et al. 2012) though social relationships during foraging are not always mediated by kinship (King et al. 2011; Madden et al. 2012). In our experiment, we regularly replaced grass patches, thereby minimizing resource depletion and mitigating the cost of food-sharing. Moreover, one or more patches were frequently unoccupied (out of a total of 6605 unique patch recordings, the patch was occupied in 33.9\% of events), suggesting that resource competition was minimal. Consequently, we expected kin / familiar individuals to associate during foraging, and this is exactly what we found. By sharing food with kin / familiar individuals, geese most likely accrued indirect fitness benefits. This effect was more pronounced in females (Fig. 4). This conforms to the observation that in the wild, female barnacle geese tend to nest close to kin (van der Jeugd et al. 2002; Anderholm et al. 2009) as opposed to males which are the dispersing sex (van der Jeugd 2001). Our results also support observations from other taxa where any fitness benefits gained from interacting with kin are much more likely to accrue to females than to males (Silk 2007; Smith et al. 2010; Wiszniewski et al. 2010; Croft et al. 2012; Silk et al. 2012).

Although barnacle geese prefer to associate with familiar individuals from earlier in their life during foraging, when choosing mates familiarity is selected against [see also Bateson (1982)]. There seem two likely and non-exclusive reasons. First, if familiarity is taken as a surrogate measure of kinship, this behaviour would reduce the risk of inbreeding (Keller \& Waller 2002). Second, if unfamiliar individuals are genetically dissimilar, this behaviour could promote outbreeding. Interestingly, our geese selected against familiarity, but there was no effect of genetic relatedness on mate choice. This may be because genetic kin recognition is unreliable: at any one gene, two full-siblings are as likely to be completely different as they are to be identical, while even multiple recognition genes would struggle to distinguish between lower levels of kinship such as between cousins. If mating opportunities 
are plentiful, a pragmatic rule of avoiding familiar individuals may be both easier to operate and highly effective. The extent to which geese have the ability to discriminate between kin and non-kin is interesting in itself. Sisters often nest close to each other, even at non-natal colonies. However, this pattern holds only for sisters born in the same year and not to sisters born in different years (van der Jeugd et al. 2002; Anderholm et al. 2009), suggesting that early life familiarity rather than ability to assess kinship could be responsible.

Although our results make good sense from the perspective of inbreeding avoidance, the finding that geese preferred to mate with unfamiliar individuals is at odds with a previous study where it was found that barnacle geese preferentially mate with familiar associates (Choudhury \& Black 1994). One possible explanation lies with the experimental set-up. In our experiment, the familiarity groups consisted of mixed-sex groups while in Choudhury and Black (1994) the familiarity groups were housed as single sex flocks that were in close proximity but did not interact directly. Combined, these results suggest that barnacle geese use their early encounters in life to identify kin, or at least a group of familiar animals who are likely to include close relatives, and then use knowledge of this group to avoid inbreeding.

Inbreeding shapes genetic variation and has major implications for the evolution of mating systems (Keller 1998). Quantifying inbreeding is, however, not without challenges. It requires the study of populations which have sufficient variation in relatedness, the correct identification of potential and actual mates, reliable estimates of relatedness and a proper statistical evaluation (Szulkin et al. 2012). These conditions are very rarely all fulfilled in empirical studies that often suffer from lack of information on whom is meeting whom and the use of pedigree information to estimate relatedness. Moreover, pedigree analysis provides expected pairwise relatedness values, but realised relatedness varies due to recombination and sampling variance (Hill \& Weir 2011). Here we used high resolution genetic markers that estimate realized relatedness to show that there is considerable variation in genetic relatedness 
among individuals in our population. The spatial proximity among individuals enabled all individuals to interact with all potentially available mates. Moreover, our experimental manipulation allowed us to differentiate between familiarity and genetic relatedness and our statistical framework allowed testing for these competing hypotheses, thereby showing inbreeding avoidance via selection against familiarity. We believe that studies akin to ours should help to elucidate the mechanisms underlying inbreeding avoidance.

\section{Personality}

There is still very little known about how personality affects assortment patterns in networks [but see (Pike et al. 2008; Krause et al. 2010)]. Dyer et al. (2008) showed that mixed shoals of bold and shy fish forage more efficiently than shoals of only bold or only shy fish. We did not find an effect of boldness on foraging associations in barnacle geese, for which we advance three non-mutually exclusive explanations. Firstly, it is possible that in foraging flocks the effect of familiarity and relatedness overrides a possible effect of boldness. A second explanation is that the environment itself was not challenging enough for boldness differences to become apparent. The foraging environment was very familiar and perhaps this assured that boldness was not of importance in making foraging decisions. It would be interesting to perform network observations while varying the degree of danger/novelty of the environment to see how this affects the role of boldness on social network structure. A third possibility is the relatively small sample size of our study especially since the strongest effects are expected at the extreme ends of the personality distribution.

We also found no evidence for a role of boldness during mate choice. Previous work suggests that parents that are more compatible in personality enjoy higher reproductive success (Dingemanse et al. 2004; Both et al. 2005; Schuett et al. 2011; Gabriel \& Black 2012) but whether individuals also actively choose 'similar' partners during mate choice is still an 
unresolved question. That we did not find an effect of boldness (or dominance) on either mating or foraging associations yet found strong effects of relatedness and familiarity suggests that neither factor should be studied in isolation. Thus, familiarity both facilitates the formation of dominance hierarchies and at the same time may be used as a cue for relatedness creating an interdependence that we were only able to resolve because both aspects were quantified. This interdependence applies particularly to social network analysis, but perhaps also to other analyses in which (dis)similarity matrices are used, such as in population genetics.

\section{Node-label permutation tests}

Social network analyses can be challenging due to the problem of non-independence of the data (James et al. 2009; Croft et al. 2011), which excludes the use of conventional statistical tests based on ordinary least squares. Randomization tests are believed to be the most robust approach to network data (Croft et al. 2011) and MRQAP is a promising tool to solve the issue of non-independence. The key prerequisite of this node-label permutation is a high reliability of the edges of the network [i.e., the network consists of interactions rather than associations, see also Croft et al. (2011)]. Technological advances (such as lighter proximity loggers, automatic tracking software) will facilitate the collection of ever more detailed and precise network data and will provide greater detail on the type of association between individuals (Krause et al. 2011). These developments will increase the reliability of the edges of networks (i.e., moving from association to interactions) and thus call for robust and flexible multivariate approaches for node-label permutations to test competing hypothesis. MRQAP is a powerful tool, as evidenced by an increase in the number of network studies that have used it (Harrison et al. 2011; Hirsch et al. 2012; Jacoby et al. 2012; Mann et al. 2012). All these 
studies used the UCINET network analysis package, which although very useful has a number of limitations.

Importantly, our framework (MRMPA) allows analysis within a generalized linear model framework, which can account for specific error distributions (binomial, Poisson or Gamma) in contrast to the widely used MRQAP in Ucinet. Additionally, it allows for constrained node-label randomizations. Constrained randomization is often a necessity in social network analysis, for example to control for temporal or spatial structure in the data. Further, by using the open source environment of $\mathrm{R}$, we hope to facilitate exchange of methods (our code is provided in Supplementary Data). Comparing MRMPA to MRQAP returned similar results, which were subsequently supported by the computed estimates and standard errors of another method, suggesting that our method is robust. However, for a full evaluation of our method a more rigorous test, including simulations is expedient. Randomizing matrices in a linear model framework is more common in other areas of ecological research (Legendre \& Fortin 2010) but has not been widely used in animal social network studies. We believe that our framework is more flexible than current methods used in animal social network analysis for node-label permutations and we hope that this will facilitate future research.

To conclude, we introduce a more flexible statistical tool for node-label permutation tests for multivariate analysis. Applying this framework to a study on barnacle geese showed contrasting context dependence of early life familiarity and genetic relatedness on social networks: barnacle geese preferentially associate with related and familiar individuals from earlier in life when foraging but select for partners that are unfamiliar during mate choice. Our results also highlight the importance of studying animal social networks across different ecological contexts. 


\section{Acknowledgements}

We thank Bart van Lith from the NIOO for his invaluable support in care taking. We are also grateful to Dick James and Tom Snijders for their statistical advice and to Richard Crooijmans, Martien Groenen, Qiong Zhang and Bert Dibbits for their help with genetic lab work, and the Animal Breeding and Genomics Group of Wageningen University for generously hosting us in their laboratories. We thank the "Faunafonds" and the "Koninklijke Nederlandse Jagers Vereniging (KNJV)" for financial support. RHJMK is funded by an NWO Rubicon Grant (825.11.014). RMJ is supported by the German Science Foundation (DFG grant KR 2089/5-1). JIH is funded by a Marie Curie Career Integration Grant (PCIG-GA2011-303618) and RHSK is funded by grant SAW-2011-SGN-3 from the Leibniz Association.

\section{References}

Anderholm, S., Waldeck, P., Van der Jeugd, H. P., Marshall, R. C., Larsson, K. \& Andersson, M. 2009. Colony kin structure and host-parasite relatedness in the barnacle goose. Molecular Ecology, 18, 4955-4963.

Aplin, L. M., Farine, D. R., Morand-Ferron, J. \& Sheldon, B. C. 2012. Social networks predict patch discovery in a wild population of songbirds. Proceedings of the Royal Society B-Biological Sciences, 279, 4199-4205.

Bateson, P. 1982. Preferences for cousins in Japanese quail. Nature, 295, 236-237.

Becker, W. A. 1984. Manual of Quantitative Genetics. Washington: Academic Enterprises, Pulman.

Black, J. M. \& Owen, M. 1989. Parent offspring relationships in wintering barnacle geese. Animal Behaviour, 37, 187-198.

Black, J. M. \& Owen, M. 1995. Reproductive performance and assortative pairing in relation to age in Barnacle geese. Journal of Animal Ecology, 64, 234-244.

Both, C., Dingemanse, N. J., Drent, P. J. \& Tinbergen, J. M. 2005. Pairs of extreme avian personalities have highest reproductive success. Journal of Animal Ecology, 74, 667674.

Choudhury, S. \& Black, J. M. 1993. Mate selection behavior and sampling strategies in geese. Animal Behaviour, 46, 747-757. 
Choudhury, S. \& Black, J. M. 1994. Barnacle geese preferentially pair with familiar associates from early-life. Animal Behaviour, 48, 81-88.

Cote, S. D. 2000. Dominance hierarchies in female mountain goats: Stability, aggressiveness and determinants of rank. Behaviour, 137, 1541-1566.

Couzin, I. D., Krause, J., Franks, N. R. \& Levin, S. A. 2005. Effective leadership and decision-making in animal groups on the move. Nature, 433, 513-516.

Croft, D. P., James, R., Thomas, P. O. R., Hathaway, C., Mawdsley, D., Laland, K. N. \& Krause, J. 2006. Social structure and co-operative interactions in a wild population of guppies (Poecilia reticulata). Behavioral Ecology and Sociobiology, 59, 644-650.

Croft, D. P., James, R. \& Krause, J. 2008. Exploring animal social networks. Princeton: Princeton University Press.

Croft, D. P., Madden, J. R., Franks, D. W. \& James, R. 2011. Hypothesis testing in animal social networks. Trends in Ecology \& Evolution, 26, 502-507.

Croft, D. P., Hamilton, P. B., Darden, S. K., Jacoby, D. M. P., James, R., Bettaney, E. M. \& Tyler, C. R. 2012. The role of relatedness in structuring the social network of a wild guppy population. Oecologia, 170, 955-963.

Cross, P. C., Lloyd-Smith, J. O., Bowers, J. A., Hay, C. T., Hofmeyr, M. \& Getz, W. M. 2004. Integrating association data and disease dynamics in a social ungulate: bovine tuberculosis in African buffalo in the Kruger National Park. Annales Zoologici Fennici, 41, 879-892.

De Vries, H. 1998. Finding a dominance order most consistent with a linear hierarchy: a new procedure and review. Animal Behaviour, 55, 827-843.

Dekker, D., Krackhardt, D. \& Snijders, T. A. B. 2007. Sensitivity of MRQAP tests to collinearity and autocorrelation conditions. Psychometrika, 72, 563-581.

Dingemanse, N. J., Both, C., Drent, P. J. \& Tinbergen, J. M. 2004. Fitness consequences of avian personalities in a fluctuating environment. Proceedings of the Royal Society $B$ Biological Sciences, 271, 847-852.

Drewe, J. A. 2010. Who infects whom? Social networks and tuberculosis transmission in wild meerkats. Proceedings of the Royal Society B-Biological Sciences, 277, 633-642.

Dyer, J. R. G., Croft, D. P., Morrell, L. J. \& Krause, J. 2008. Shoal composition determines foraging success in the guppy. Behavioral Ecology, 20, 165-171.

Efron, B. \& Tibshirani, R. 1994. An Introduction to the Bootstrap: Chapman and Hall, New York.

Farine, D. R., Garroway, C. J. \& Sheldon, B. C. 2012. Social network analysis of mixedspecies flocks: exploring the structure and evolution of interspecific social behaviour. Animal Behaviour, 84, 1271-1277.

Flack, J. C., Girvan, M., de Waal, F. B. M. \& Krakauer, D. C. 2006. Policing stabilizes construction of social niches in primates. Nature, 439, 426-429.

Forkman, B. \& Haskell, M. J. 2004. The maintenance of stable dominance hierarchies and the pattern of aggression: Support for the suppression hypothesis. Ethology, 110, 737744. 
Foster, E. A., Franks, D. W., Morrell, L. J., Balcomb, K. C., Parsons, K. M., van Ginneken, A. \& Croft, D. P. 2012. Social network correlates of food availability in an endangered population of killer whales, Orcinus orca. Animal Behaviour, 83, 731-736.

Franks, D. W., Ruxton, G. D. \& James, R. 2010. Sampling animal association networks with the gambit of the group. Behavioral Ecology and Sociobiology, 64, 493-503.

Gabriel, P. O. \& Black, J. M. 2012. Behavioural syndromes, partner compatibility and reproductive performance in steller's jays. Ethology, 118, 76-86.

Ginsberg, J. R. \& Young, T. P. 1992. Measuring association between individuals or groups in behavioral studies. Animal Behaviour, 44, 377-379.

Guttal, V. \& Couzin, I. D. 2010. Social interactions, information use, and the evolution of collective migration. Proceedings of the National Academy of Sciences of the United States of America, 107, 16172-16177.

Hamilton, W. D. 1964. The genetical evolution of social behaviour. II. Journal of Theoretical Biology, 7, 17-52.

Harrison, F., Sciberras, J. \& James, R. 2011. Strength of Social Tie Predicts Cooperative Investment in a Human Social Network. PLoS ONE, 6, e18338.

Henzi, S. P., Lusseau, D., Weingrill, T., Schaik, C. P. \& Barrett, L. 2009. Cyclicity in the structure of female baboon social networks. Behavioral Ecology and Sociobiology, 63, 1015-1021.

Hill, W. G. \& Weir, B. S. 2011. Variation in actual relationship as a consequence of Mendelian sampling and linkage. Genetics Research, 93, 47-64.

Hirsch, B. T., Stanton, M. A. \& Maldonado, J. E. 2012. Kinship Shapes Affiliative Social Networks but Not Aggression in Ring-Tailed Coatis. PLoS ONE, 7.

Hobson, E. A., Avery, M. L. \& Wright, T. F. 2013. An analytical framework for quantifying and testing patterns of temporal dynamics in social networks. Animal Behaviour, 85, 83-96.

Holekamp, K. E., Smith, J. E., Strelioff, C. C., Van Horn, R. C. \& Watts, H. E. 2012. Society, demography and genetic structure in the spotted hyena. Molecular Ecology, 21, 613-632.

Jacoby, D. M. P., Brooks, E. J., Croft, D. P. \& Sims, D. W. 2012. Developing a deeper understanding of animal movements and spatial dynamics through novel application of network analyses. Methods in Ecology and Evolution, 3, 574-583.

James, R., Croft, D. P. \& Krause, J. 2009. Potential banana skins in animal social network analysis. Behavioral Ecology and Sociobiology, 63, 989-997.

Jonker, R. M., Zhang, Q., Van Hooft, P., Loonen, M., Van der Jeugd, H. P., Crooijmans, R., Groenen, M. A. M., Prins, H. H. T. \& Kraus, R. H. S. 2012. The development of a genome wide SNP set for the barnacle goose Branta leucopsis. PLoS ONE, 7, e38412.

Keller, L. F. 1998. Inbreeding and its fitness effects in an insular population of song sparrows (Melospiza melodia). Evolution, 52, 240-250.

Keller, L. F. \& Waller, D. M. 2002. Inbreeding effects in wild populations. Trends in Ecology \& Evolution, 17, 230-241. 
King, A. J., Clark, F. E. \& Cowlishaw, G. 2011. The dining etiquette of desert baboons: the roles of social bonds, kinship, and dominance in co-feeding networks. American Journal of Primatology, 73, 768-774.

Krackhardt, D. 1988. Predicting with networks: Nonparametric multiple regression analysis of dyadic data. Social Networks, 10, 359-381.

Kraus, R. H. S., Kerstens, H., Van Hooft, P., Crooijmans, R., Van Der Poel, J., Elmberg, J., Vignal, A., Huang, Y., Li, N., Prins, H. \& Groenen, M. 2011. Genome wide SNP discovery, analysis and evaluation in mallard (Anas platyrhynchos). BMC Genomics, 12, 150 .

Krause, J., Croft, D. P. \& James, R. 2007. Social network theory in the behavioural sciences: potential applications. Behavioral Ecology and Sociobiology, 62, 15-27.

Krause, J., Lusseau, D. \& James, R. 2009. Animal social networks: an introduction. Behavioral Ecology and Sociobiology, 63, 967-973.

Krause, J., James, R. \& Croft, D. P. 2010. Personality in the context of social networks. Philosophical Transactions of the Royal Society B-Biological Sciences, 365, 4099-4106.

Krause, J., Wilson, A. D. M. \& Croft, D. P. 2011. New technology facilitates the study of social networks. Trends in Ecology \& Evolution, 26, 5-6.

Kurvers, R. H. J. M., Eijkelenkamp, B., van Oers, K., van Lith, B., van Wieren, S. E., Ydenberg, R. C. \& Prins, H. H. T. 2009. Personality differences explain leadership in barnacle geese. Animal Behaviour, 78, 447-453.

Kurvers, R. H. J. M., Prins, H. H. T., van Wieren, S. E., van Oers, K., Nolet, B. A. \& Ydenberg, R. C. 2010. The effect of personality on social foraging: shy barnacle geese scrounge more. Proceedings of the Royal Society B-Biological Sciences, 277, 601-608.

Kurvers, R. H. J. M., Adamczyk, V. M. A. P., van Wieren, S. E. \& Prins, H. H. T. 2011. The effect of boldness on decision-making in barnacle geese is group-size-dependent. Proceedings of the Royal Society Biological Sciences Series B, 278, 2018-2024.

Kurvers, R. H. J. M., de Hoog, S. I. V., van Wieren, S. E., Ydenberg, R. C. \& Prins, H. H. T. 2012a. No evidence for negative frequency-dependent feeding performance in relation to personality. Behavioral Ecology, 23, 51-57.

Kurvers, R. H. J. M., Nolet, B. A., Prins, H. H. T., Ydenberg, R. C. \& van Oers, K. 2012b. Boldness affects foraging decisions in barnacle geese: an experimental approach. Behavioral Ecology, 23, 1155-1161.

Legendre, P. \& Fortin, M. J. 2010. Comparison of the Mantel test and alternative approaches for detecting complex multivariate relationships in the spatial analysis of genetic data. Molecular Ecology Resources, 10, 831-844.

Lessells, C. M. \& Boag, P. T. 1987. Unrepeatable repeatabilities - a common mistake. Auk, 104, 116-121.

Lusseau, D., Barrett, L. \& Henzi, S. P. 2011. Formalising the multidimensional nature of social networks. arXiv, 1101.3735v1.

Madden, J. R., Nielsen, J. F. \& Clutton-Brock, T. H. 2012. Do networks of social interactions reflect patterns of kinship? Current Zoology, 58, 319-328. 
Mann, J., Stanton, M. A., Patterson, E. M., Bienenstock, E. J. \& Singh, L. O. 2012. Social networks reveal cultural behaviour in tool-using using dolphins. Nature Communications, 3, 980.

Mathot, K. J. \& Giraldeau, L. A. 2010. Within-group relatedness can lead to higher levels of exploitation: a model and empirical test. Behavioral Ecology, 21, 843-850.

Milligan, B. G. 2003. Maximum-likelihood estimation of relatedness. Genetics, 163, 11531167.

Pike, T. W., Samanta, M., Lindstrom, J. \& Royle, N. J. 2008. Behavioural phenotype affects social interactions in an animal network. Proceedings of the Royal Society BBiological Sciences, 275, 2515-2520.

Schuett, W., Tregenza, T. \& Dall, S. R. X. 2010. Sexual selection and animal personality. Biological Reviews, 85, 217-246.

Schuett, W., Dall, S. R. X. \& Royle, N. J. 2011. Pairs of zebra finches with similar 'personalities' make better parents. Animal Behaviour, 81, 609-618.

Sih, A., Hanser, S. F. \& McHugh, K. A. 2009. Social network theory: new insights and issues for behavioral ecologists. Behavioral Ecology and Sociobiology, 63, 975-988.

Silk, J. B. 2007. Social components of fitness in primate groups. Science, 317, 1347-1351.

Silk, J. B., Alberts, S. C., Altmann, J., Cheney, D. L. \& Seyfarth, R. M. 2012. Stability of partner choice among female baboons. Animal Behaviour, 83, 1511-1518.

Smith, J. E., Van Horn, R. C., Powning, K. S., Cole, A. R., Graham, K. E., Memenis, S. K. \& Holekamp, K. E. 2010. Evolutionary forces favoring intragroup coalitions among spotted hyenas and other animals. Behavioral Ecology, 21, 284-303.

Szulkin, M., Stopher, K. V., Pemberton, J. M. \& Reid, J. M. 2012. Inbreeding avoidance, tolerance, or preference in animals? Trends in Ecology \& Evolution, 28, 205-211.

van der Jeugd, H. P. 2001. Large barnacle goose males can overcome the social costs of natal dispersal. Behavioral Ecology, 12, 275-282.

van der Jeugd, H. P. \& Blaakmeer, K. B. 2001. Teenage love: the importance of trial liaisons, subadult plumage and early pairing in barnacle geese. Animal Behaviour, 62, 1075-1083.

van der Jeugd, H. P., van der Veen, I. T. \& Larsson, K. 2002. Kin clustering in barnacle geese: familiarity or phenotype matching? Behavioral Ecology, 13, 786-790.

Wang, J. L. 2011. COANCESTRY: a program for simulating, estimating and analysing relatedness and inbreeding coefficients. Molecular Ecology Resources, 11, 141-145.

Wey, T., Blumstein, D. T., Shen, W. \& Jordan, F. 2008. Social network analysis of animal behaviour: a promising tool for the study of sociality. Animal Behaviour, 75, 333-344.

Wey, T. W. \& Blumstein, D. T. 2012. Social attributes and associated performance measures in marmots: bigger male bullies and weakly affiliating females have higher annual reproductive success. Behavioral Ecology and Sociobiology, 66, 1075-1085.

Whitehead, H. 2008. Analyzing animal societies: Quantative methods for vertebrate social analysis. Chicago: University of Chicago Press.

Whitehead, H. 2009. SOCPROG programs: analysing animal social structures. Behavioral Ecology and Sociobiology, 63, 765-778. 
662

663

664

665

666

667

668

669

Wiszniewski, J., Lusseau, D. \& Moller, L. M. 2010. Female bisexual kinship ties maintain social cohesion in a dolphin network. Animal Behaviour, 80, 895-904.

Wiszniewski, J., Brown, C. \& Moller, L. M. 2012. Complex patterns of male alliance formation in a dolphin social network. Journal of Mammalogy, 93, 239-250.

The following Supporting Information is available for this article online:

Supplementary Data. R code of MRMPA method. 
Table 1. The effect of familiarity, relatedness, boldness and dominance on the female

671 foraging associations, male foraging associations and pair formation. Shown are the results of 672 the MRQAP method and the MRMPA method.

673

\begin{tabular}{ccccccc}
\hline Females & \multicolumn{3}{c}{ MRQAP } & \multicolumn{3}{c}{ MRMPA } \\
\cline { 2 - 7 } & Est & SE & $P$-value & Est & SE & $P$-value \\
Familiarity & 0.037 & 0.007 & 0.0001 & 0.036 & 0.006 & $<0.0001$ \\
Relatedness & 0.446 & 0.051 & 0.0001 & 0.452 & 0.041 & $<0.0001$ \\
Boldness & 0.007 & 0.005 & 0.073 & 0.006 & 0.004 & 0.165 \\
Dominance & 0.0000 & 0.0000 & 0.45 & 0.00003 & 0.0006 & 0.904 \\
\hline Males & & & & & & \\
& & & & & & \\
Familiarity & 0.015 & 0.007 & 0.030 & 0.015 & 0.007 & 0.043 \\
Relatedness & 0.183 & 0.042 & 0.002 & 0.180 & 0.041 & 0.002 \\
Boldness & 0.0008 & 0.005 & 0.45 & 0.0009 & 0.005 & 0.876 \\
Dominance & 0.0004 & 0.0004 & 0.27 & 0.0004 & 0.0006 & 0.517 \\
\hline Mate choice & & & & & & \\
Familiarity & & & & -1.0360 & 0.2389 & 0.030 \\
Relatedness & & & & 0.1731 & 0.1425 & 0.59 \\
Boldness & & & & & & \\
\hline
\end{tabular}

674 
677

678

679

680

681

682

683

684

685

686

687

688

689

690

691

692

693

694

695

696

697

698

\section{List of figures}

Figure 1. Boxplot showing the pairwise genetic relatedness of dyads from the same familiarity groups ("familiar") and of dyads from the different familiarity groups ("unfamiliar"). Box plots show the median value (horizontal line), the interquartile range (25 $-75 \%$ ) (box), the maximum value excluding outliers (whisker) and the outliers (dots).

Figure 2. Complete female (a, circles) and male (b, squares) foraging networks. Grey lines represent weak connections $(\mathrm{SRI}<0.1)$ and black lines represent strong connections $(\mathrm{SRI} \geq$ 0.1). The width of the black lines indicates the strength of the association (thin black line: 0.1 $\leq \mathrm{SRI}<0.2$, medium black line: $0.2 \leq \mathrm{SRI}<0.4$, thick black line: $\mathrm{SRI} \geq 0.4$ ).

Figure 3. Filtered (SRI $\geq 0.1$ ) female (a, circles) and male (b, squares) foraging networks. Black lines represent strong foraging associations $(\mathrm{SRI} \geq 0.1)$ and grey lines represent strongly genetically related individuals (relatedness > 0.4). Black dots/squares represent individuals that originate from familiarity group 1; grey dots/squares represent geese that originate from familiarity group 2.

Figure 4. Effect sizes \pm S.E. of the effect of familiarity, genetic relatedness, boldness and dominance on the female (triangles) and male (circles) foraging associations and on mate choice (squares). Left y-axis corresponds to the effect sizes of the variables during the foraging associations (triangles and circles). Right y-axis corresponds to the effect sizes of the variables during mate choice (squares). 


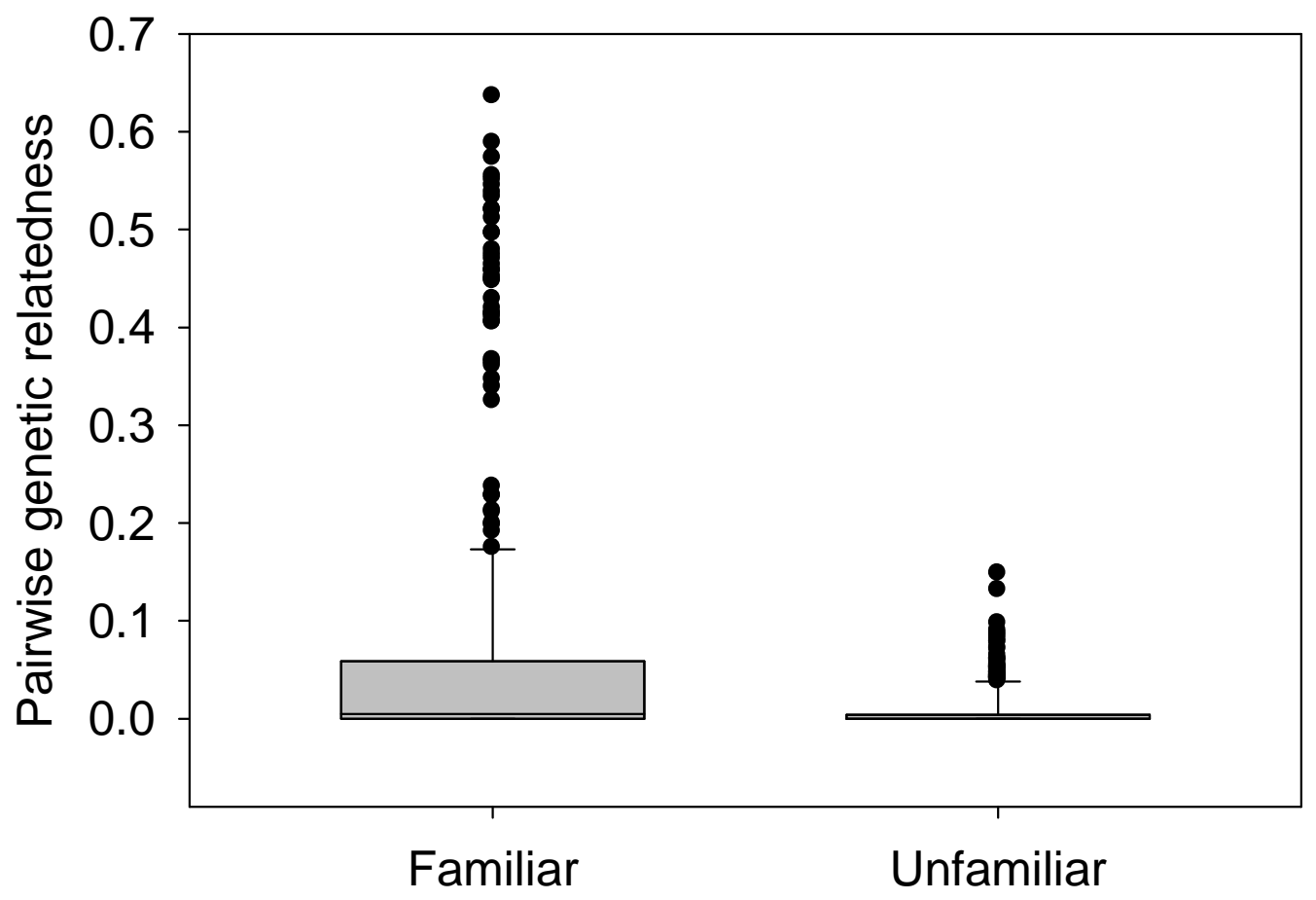


$702 \quad$ Figure 2

(a)
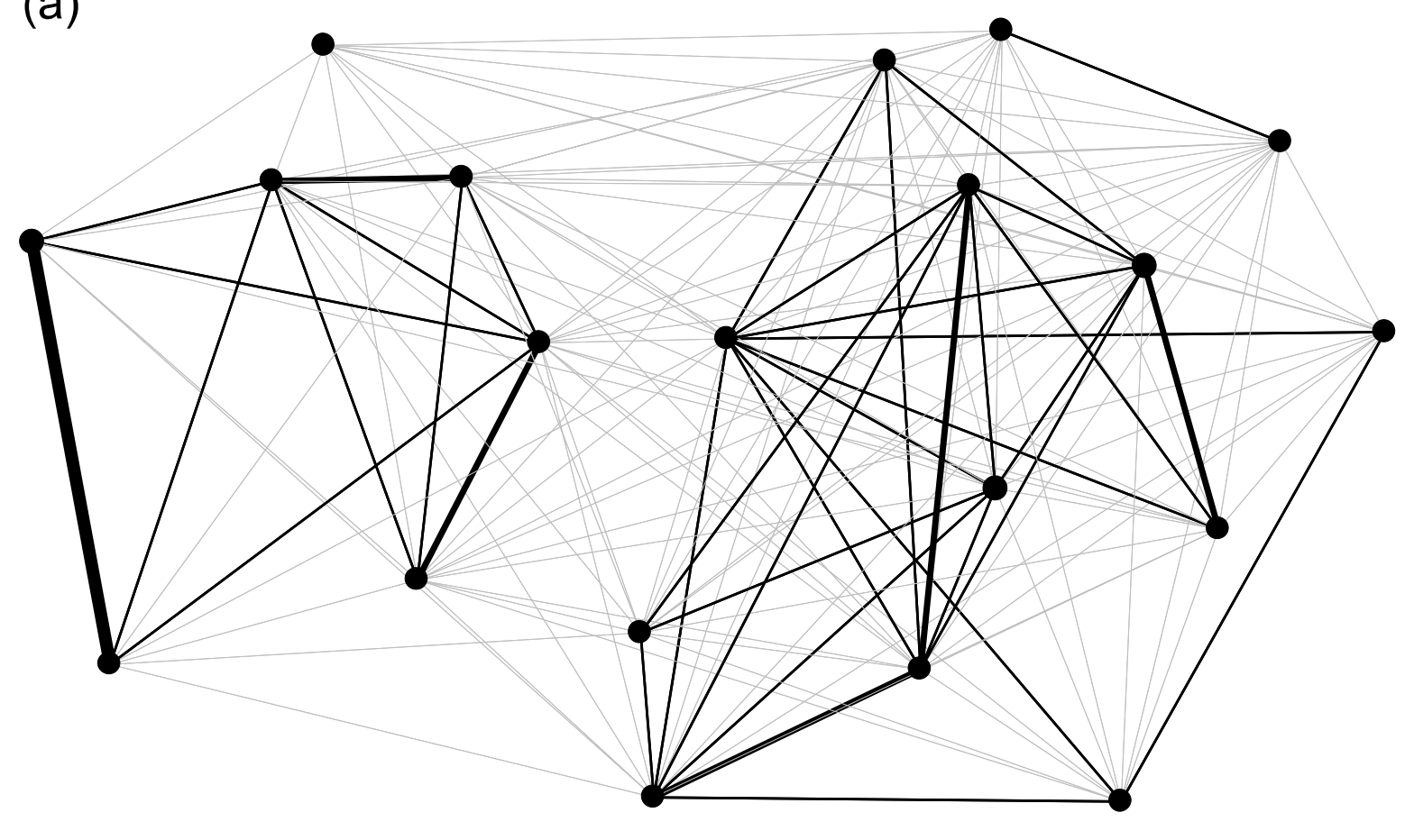

703

(b)

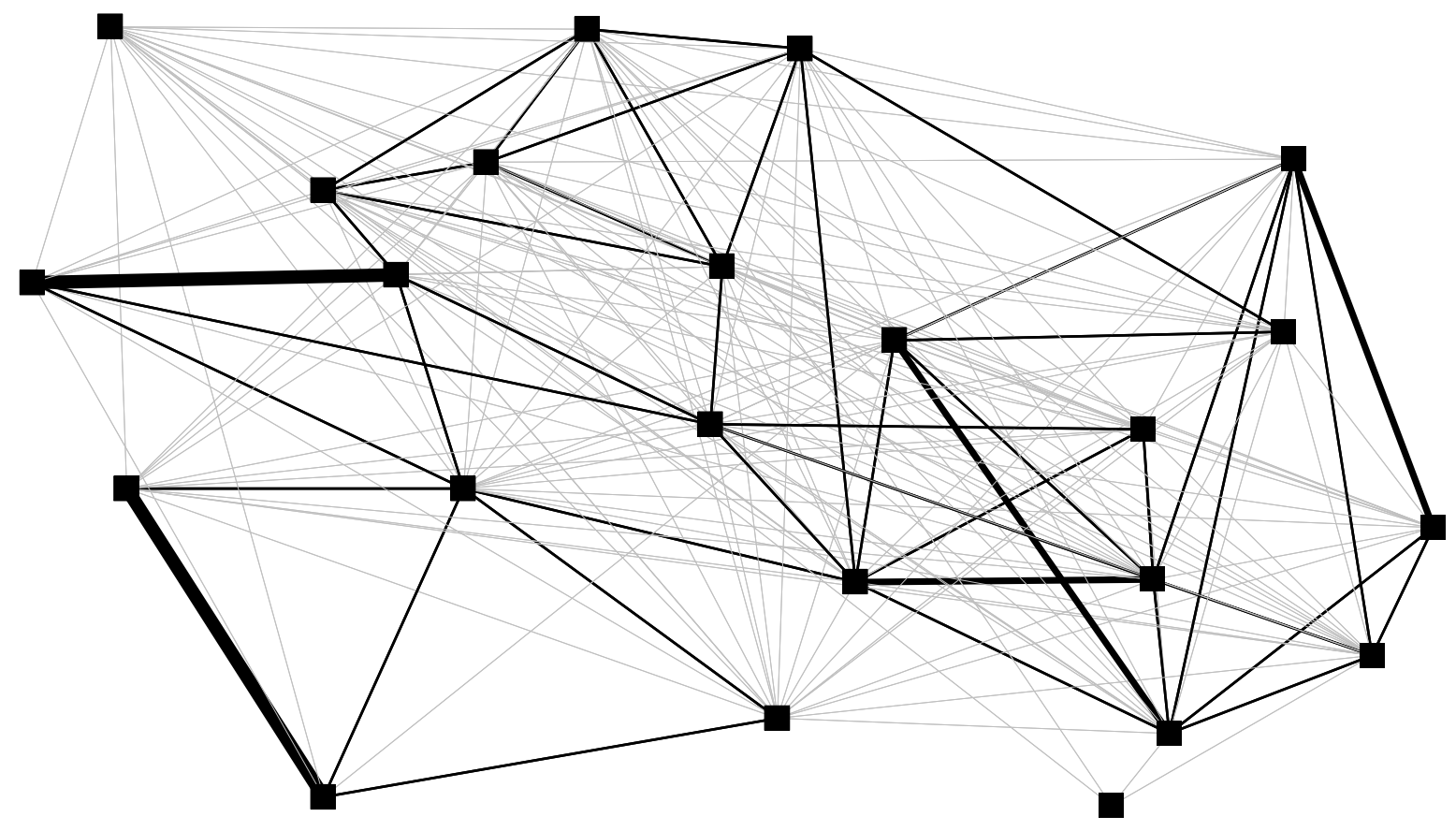


$705 \quad$ Figure 3

(a)
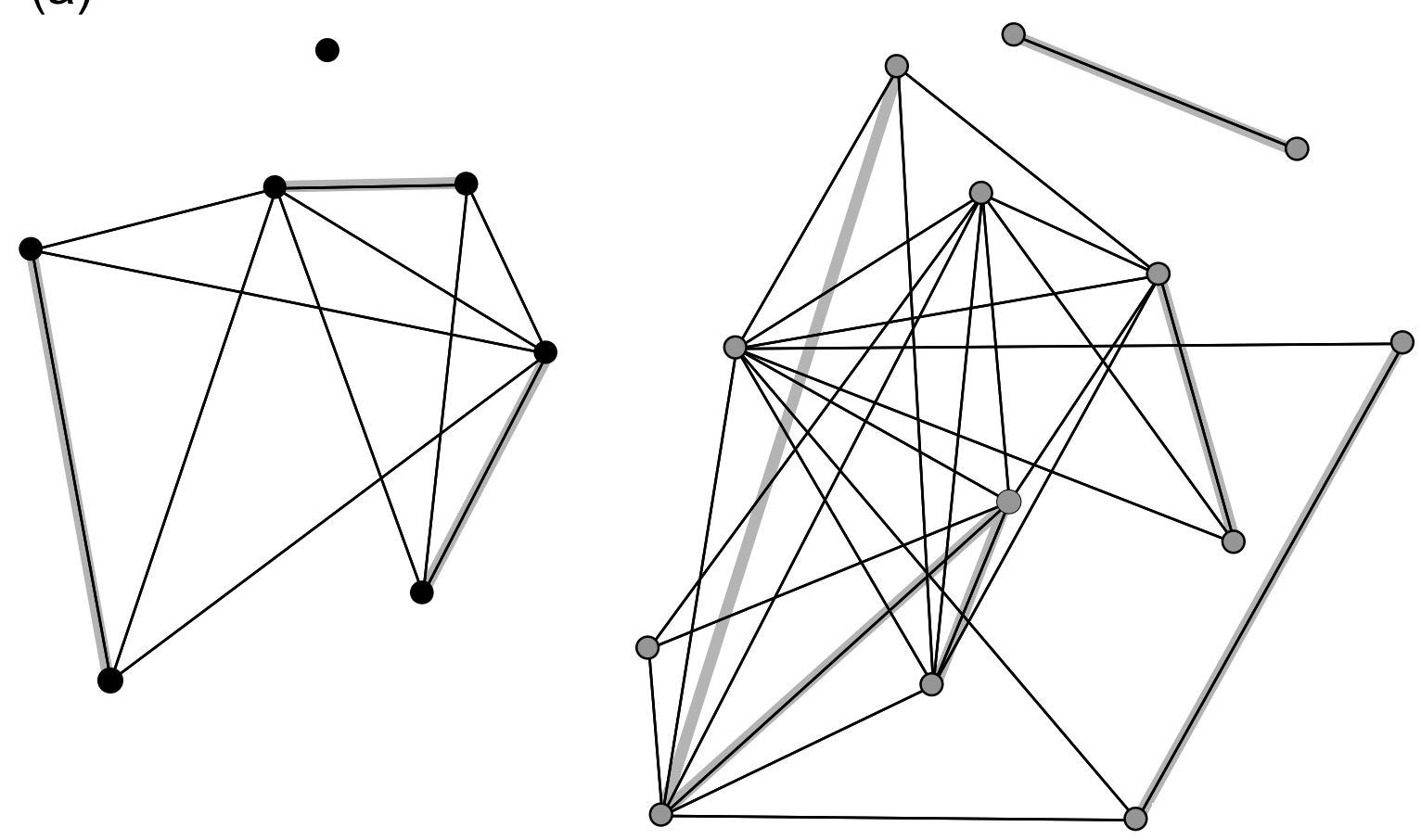

706

(b)

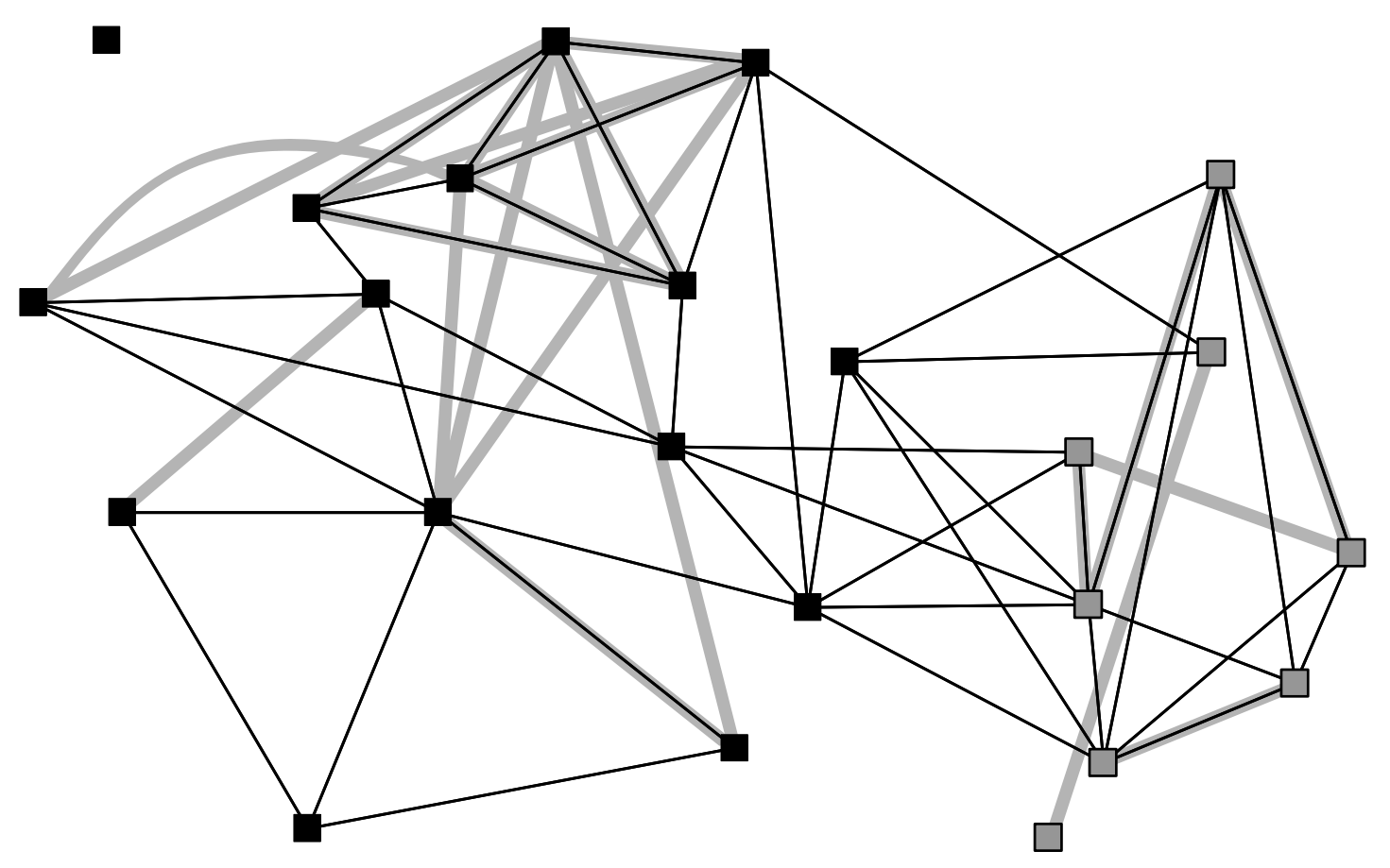




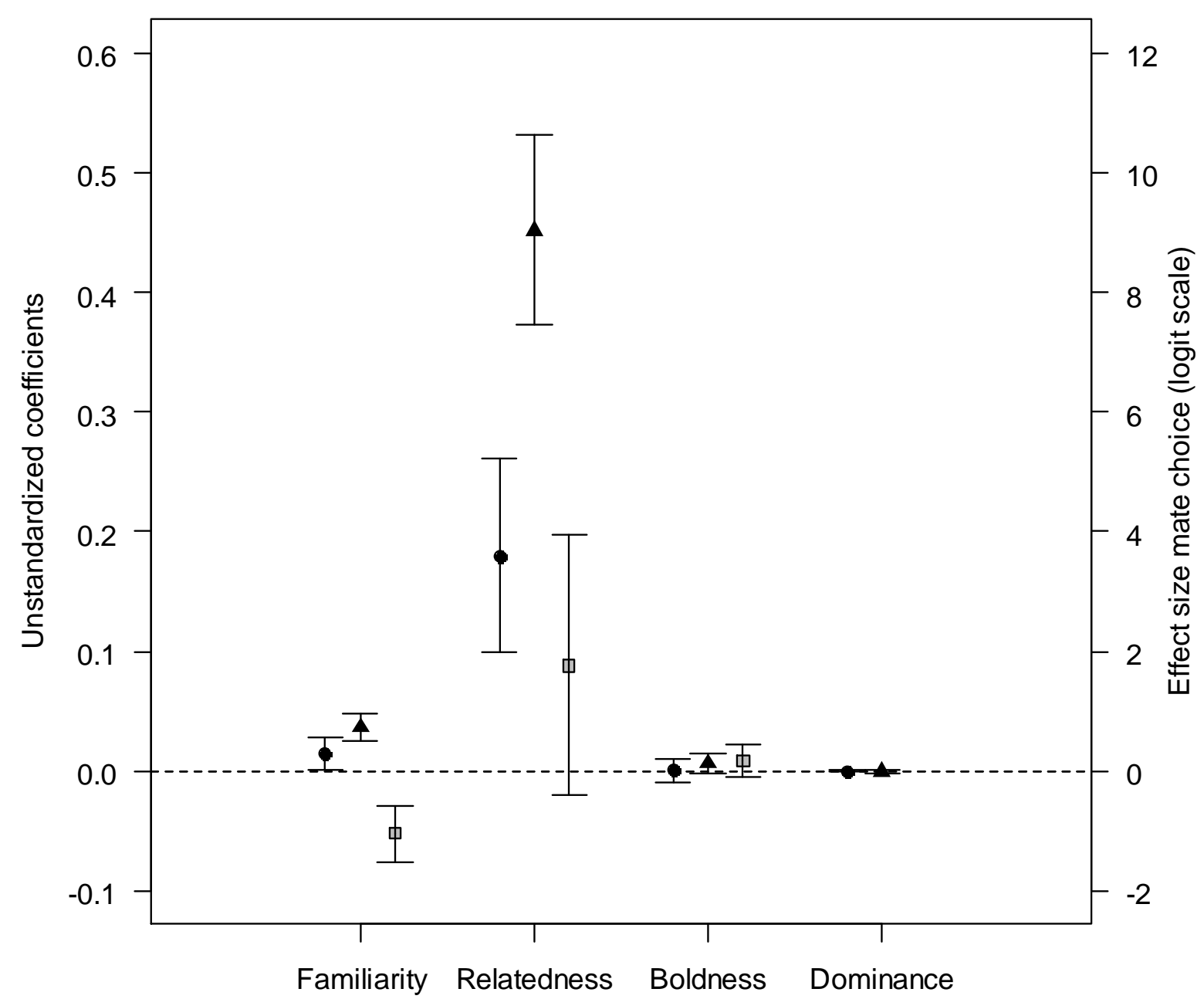

Variable 


\begin{tabular}{|c|c|c|c|c|c|c|c|c|c|c|c|c|c|c|c|c|c|c|c|c|}
\hline$\checkmark$ & 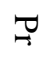 & $=$ & 즈 & $\leftrightarrows$ & $\stackrel{\mathbb{I}}{\exists}$ & $\Omega$ & $\Omega$ & $\underset{G}{\mathscr{G}}$ & $\underline{\sigma}$ & 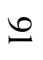 & 9 & $\ddot{r}$ & $\ddot{u}$ & \pm & $\simeq$ & $\stackrel{N}{G}$ & $\stackrel{\sim}{\sigma}$ & $F$ & $\bar{\sigma}$ & \\
\hline$a$ & $\underset{\omega}{w}$ & - & $a$ & $\vec{\sigma}$ & 0 & 0 & $v$ & 6 & $\vec{D}$ & $\vec{a}$ & $\Delta$ & $\bar{N}$ & $\omega$ & $\Delta$ & 0 & $\infty$ & 6 & $a$ & $\stackrel{8}{\perp}$ & $\vec{G}$ \\
\hline$\infty$ & 0 & 0 & 0 & - & - & 0 & $N$ & 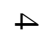 & $\omega$ & N & 0 & 二 & - & 0 & $\vec{a}$ & $\infty$ & 0 & $\stackrel{\infty}{0}$ & $a$ & $F$ \\
\hline$N$ & 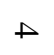 & uు & 6 & N & $N$ & $\vec{b}$ & $N$ & $a$ & $\beth$ & $a$ & $\infty$ & $a$ & 二 & $N$ & $N$ & $N$ & N & 0 & 0 & $\underline{\tilde{\sigma}}$ \\
\hline 8 & $\overline{0}$ & $\omega$ & $N$ & - & $N$ & $N$ & 0 & $\omega_{\infty}$ & $a$ & $\stackrel{N}{\perp}$ & 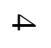 & ă & 0 & - & ニ & $\vec{a}$ & $N$ & $\infty$ & $\infty$ & $\underset{\sim}{N}$ \\
\hline$\omega$ & ur & 0 & $N$ & 0 & - & 0 & $N$ & $u$ & $N$ & च & 0 & 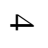 & 0 & 0 & 9 & ニ & $N$ & $\vec{a}$ & 0 & $\underline{N}$ \\
\hline - & $a$ & 0 & $N$ & $\Delta$ & $N$ & - & $\vec{\infty}$ & $A$ & - & - & 0 & ur & - & $\stackrel{t}{a}$ & 0 & - & $N$ & 0 & $\perp$ & 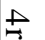 \\
\hline- & $\omega$ & - & 0 & $\perp$ & 0 & $a$ & $N$ & 0 & $\infty$ & $\perp$ & $\checkmark$ & - & 9 & - & 0 & 0 & 二 & - & $\omega$ & 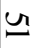 \\
\hline A & $\omega$ & 0 & $\bar{u}$ & $\checkmark$ & $\checkmark$ & 0 & $a$ & $\omega$ & 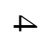 & $\stackrel{\omega}{\perp}$ & ur & $\vec{a}$ & - & ur & 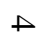 & z̆ & $a$ & 二 & $\bar{N}$ & $y$ \\
\hline$\oplus$ & 0 & $\vec{u}$ & $a$ & ur & - & $\Xi$ & $N$ & $N$ & $\Xi$ & 0 & $\overrightarrow{\text { ŭ }}$ & ur & $\checkmark$ & 0 & 0 & $\Delta$ & $N$ & 0 & $\triangle$ & 9 \\
\hline 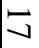 & $\stackrel{\overrightarrow{ }}{+}$ & $N$ & $\bar{N}$ & $\checkmark$ & $\omega$ & 0 & $\overline{0}$ & V & $\perp$ & $\vec{a}$ & 0 & $\stackrel{\omega}{\perp}$ & 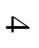 & - & $\Xi$ & $\stackrel{N}{\perp}$ & $a$ & $\stackrel{N}{\omega}$ & $\vec{a}$ & 0 \\
\hline 0 & $\infty$ & $\vec{\sigma}$ & $\omega$ & $\stackrel{N}{\simeq}$ & - & $\overline{0}$ & $N$ & $\checkmark$ & $\vec{\infty}$ & $\perp$ & $\Xi$ & 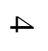 & $\infty$ & - & $N$ & $a$ & $\beth$ & $\omega$ & $\vec{A}$ & $\varpi$ \\
\hline w & $\varpi$ & 0 & $\tilde{\sigma}$ & ur & $\omega$ & 0 & $\overrightarrow{0}$ & $\overrightarrow{\vec{r}}$ & $\checkmark$ & $\nabla$ & $N$ & $\omega$ & 0 & $\triangle$ & ur & $w_{\infty}$ & $a$ & $\triangle$ & 6 & $\varpi$ \\
\hline$\Delta$ & $\omega$ & - & $\overrightarrow{0}$ & $\omega$ & $a$ & 0 & $\grave{o}$ & $\overrightarrow{0}$ & $N$ & $\overrightarrow{0}$ & $N$ & $a$ & $N$ & $\vec{\infty}$ & $N$ & 0 & $N$ & $N$ & $\checkmark$ & $\Omega$ \\
\hline 0 & 0 & w & 0 & 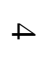 & $N$ & $\vec{r}$ & 0 & 0 & $\overrightarrow{0}$ & 0 & $\Xi$ & 0 & $a$ & - & 0 & $N$ & $\vec{b}$ & 0 & 0 & $\Omega$ \\
\hline 二 & u & 0 & $\checkmark$ & ur & 元 & $N$ & $a$ & $\omega$ & - & $\omega$ & - & $\checkmark$ & 0 & $N$ & - & $N$ & $N$ & - & 0 & 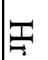 \\
\hline$\checkmark$ & $\vec{\omega}$ & 0 & ur & $\vec{u}$ & ur & $A$ & $\omega$ & $u$ & $\stackrel{N}{\sim}$ & $\checkmark$ & 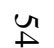 & $\checkmark$ & $\Delta$ & $A$ & 0 & - & $N$ & - & $\ddot{0}$ & $=$ \\
\hline$\infty$ & $\overrightarrow{0}$ & $N$ & $\vec{\infty}$ & ur & $\checkmark$ & 0 & $\overrightarrow{0}$ & Na & $\omega$ & $\vec{N}$ & $a$ & $\bar{u}$ & 0 & $N$ & $N$ & N & 0 & 0 & $a$ & $\mathbb{\Delta}$ \\
\hline b & - & $\vec{a}$ & $N$ & 6 & 0 & ڤె & - & 0 & $\overline{0}$ & $N$ & $\vec{u}$ & 0 & - & 0 & 0 & $\omega$ & $\ddot{\infty}$ & 0 & - & $F$ \\
\hline$\infty$ & $\vec{\infty}$ & - & $\overrightarrow{0}$ & $\bar{\omega}$ & $\underline{u}$ & 0 & $\omega$ & $\vec{\infty}^{\prime}$ & $\infty$ & $\stackrel{\vec{A}}{ }$ & 0 & $\omega$ & $\omega$ & $a$ & ur & $\overrightarrow{0}$ & $\triangle$ & 6 & $\omega$ & 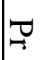 \\
\hline $\bar{r}$ & $\vec{\infty}$ & 0 & $\infty$ & $\checkmark$ & 二 & 0 & $A$ & $\underset{\omega}{\omega}$ & 0 & $\Xi$ & $A$ & $\stackrel{A}{N}$ & 一 & 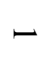 & $\omega$ & $b$ & $N$ & $\infty$ & $a$ & $\approx$ \\
\hline
\end{tabular}

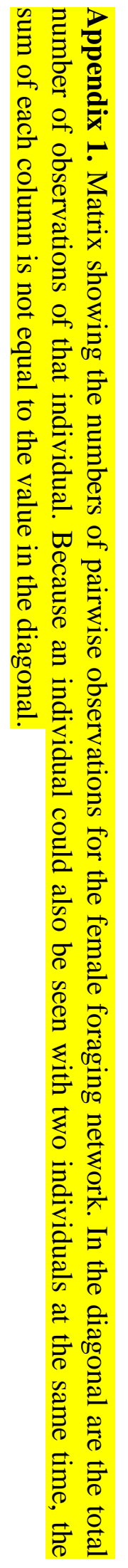



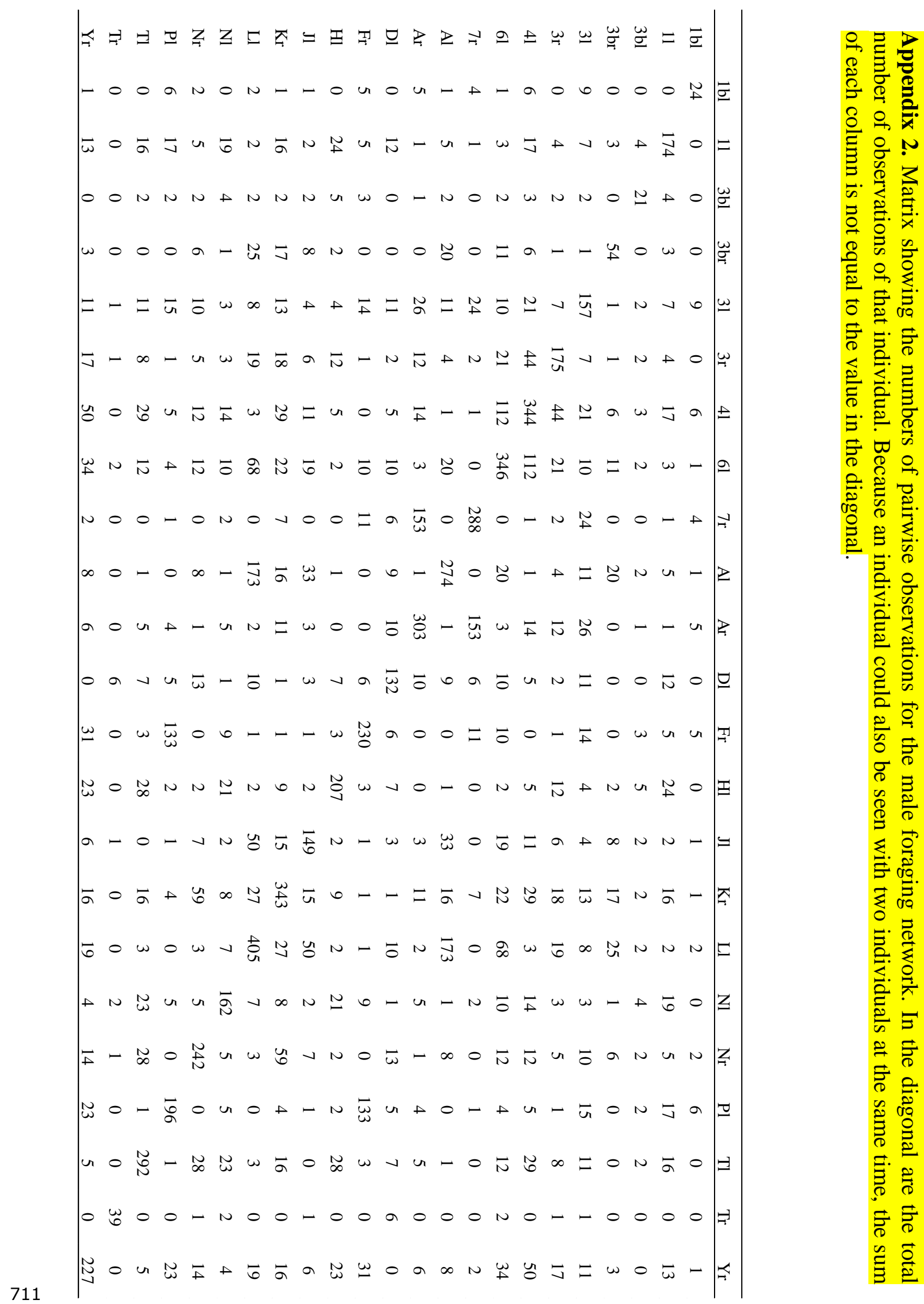